\title{
GANAR TAMAÑO Y TERRITORIO: FUSIONES DE EMPRESAS Y NUEVOS YACIMIENTOS DE ACTIVIDAD EN EL SECTOR INMOBILIARIO ESPAÑOL
}

\author{
Basilio Calderón Calderón \\ Departamento de Geografía \\ Universidad de Valladolid
}

\section{RESUMEN}

Desde el año 1995, las empresas inmobiliarias españolas han transformado profundamente la habitual estrategia del negocio inmobiliario. Los rasgos del último lustro del siglo XX están presididos por las compras y fusiones de empresas, avaladas frecuentemente por grupos bancarios, como fórmula para competir en un mercado progresivamente más abierto y más exigente, por la reducción de su negocio tradicional — la promoción de vivienda - y por el aumento del peso de otras actividades más seguras y rentables como son la gestión de alquileres —especialmente de oficinas-y la formación de reservas estratégicas de suelo. Y todas estas estrategias se desarrollan no sólo en la ciudad en la que la empresa tiene su sede social sino también en mercados regionales, nacionales e internacionales.

Palabras clave: Sector inmobiliario, fusión de empresas, alquiler de oficinas y SBA en centros comerciales.

\begin{abstract}
Spanish building societies have deeply changed their usual strategies in relation to the real-estate business since 1995. The main features of the real-estate market during the last five years of the twentieth century were characterized by the purchase and amalgamation of enterprises, which were frequently endorsed by banking systems as the only way to compete in a very open and demanding market, by the reduction in their traditional business - housing promotion - and by the increase in other safer and more profitable activities such as rent management - particularly offices - and strategic reserves of housing-estate land. All these strategies are developed not only in the city in which the company has its head office but also in regional, national and international markets.
\end{abstract}

Key words: Real-estate sector, amalgamation of enterprises, office-rent, shopingcentre-rent. 
En los últimos años del siglo XX y primeros meses del siglo XXI ha tenido lugar un desarrollo espectacular del sector de la promoción inmobiliaria, que viene a romper con alguna de las limitaciones estructurales que caracterizaba a un sector de actividad que aporta casi el 3 por 100 del PIB español; el sostenido e imparable incremento del precio de las viviendas, la necesidad de invertir para blanquear dinero antes de la entrada en vigor del Euro en el año 2002, el escaso rendimiento de las inversiones alternativas y el brusco hundimiento bursátil del sector de las telecomunicaciones - sector de moda en el último quinquenio del siglo pasado-, ha dado lugar a un periodo de bonanza en la actividad constructiva sin precedentes, acentuando el carácter de sector refugio y fiscalmente opaco que tradicionalmente ha tenido la propiedad y gestión inmobiliaria en España. Prueba de ello es el hecho de que, desde el comienzo del ciclo expansivo del mercado inmobiliario en 1997, se han iniciado en España un total de casi 2 millones de viviendas que, considerando sus valores de tasación medios, han supuesto más de 24,2 billones de pesetas de inversión en nueva vivienda por parte de las familias; es decir, una media que supera los 6 billones de inversión anual, solo en viviendas nuevas ${ }^{1}$. Como era de esperar, este volumen de actividad ha estimulado la aparición de cientos de pequeñas empresas oportunistas, vinculadas de forma más o menos directa al sector inmobiliario, que aprovechan la coyuntura y vienen a acentuar su tradicional minifundio empresarial; pero, al mismo tiempo, ha servido para que algunas grandes empresas hayan tomado conciencia de sus limitaciones, en especial de su pequeño tamaño en relación con las empresas dominantes en la Unión Europea, desplegando diversas iniciativas para ganarlo por medio de las fusiones o adquisiciones y mejorar con ello su posición relativa en la Unión Europea; conviene no olvidar que las inmobiliarias españolas de entidad, es decir, realmente competitivas, son pocas y no tienen prácticamente liquidez como sector, por lo que, en realidad. «... son un cero a la izquierda en las carteras de los inversores internacionales»².

Además de razones de carácter estructural, como las relacionadas con las obligaciones que impone la Ley Orgánica de la Edificación en materia de garantías y seguros, la coyuntura para desarrollar estas iniciativas empresariales era - y lo es aún-idónea, ya que prácticamente todas las empresas vienen repitiendo, semestre tras semestre, beneficios históricos; a modo de ejemplo sólo en los seis primeros meses del año 2001, la inmobiliaria Metrovacesa incrementó su beneficio en un 31 por 100, y espera cerrar en año en torno al 25 por 100, Vallehermoso mejoró su beneficio en un 48,5 al calor del espectacular incremento de la edificación residencial, la inmobiliaria Urbis lo hizo en un 71 por 100, en tanto que, Ferrovial Inmobiliaria aumentó sus beneficios entre Enero y Junio de 2001 en un 249,3 por 100 , por señalar tan sólo algunos ejemplos del dinamismo de este sector ${ }^{3}$.

No es extraño que, en este contexto, el banco de inversión estadounidense Merrill Lynch elevase en Julio de 2001 sus previsiones sobre las inmobiliarias españolas; bastaba con observar el comportamiento bursátil de las cuatro mayores compañías que operan en el mercado continuo: las acciones de Metrovacesa subieron en el primer semestre de 2001 un 15,06 por 100, las de Vallehermoso un 14,04 y si Inmobiliaria Colonial perdió un 3,15 , para el citado banco es una garantía la seguridad y solidez de la cartera de oficinas que esta inmobiliaria posee en Madrid y Barcelona. Finalmente, Urbis, que también perdió en bolsa un 1,26 por 100 asegura una rápida recuperación a la vista del éxito de su

1 Servicio de estudios del BBVA. Situación inmobiliaria, Febrero 2001.

2 http://www.inmonoticias.com/entrevis/ENTRE_basagoiti.htm

3 Las inmobiliarias, en la cresta de la ola. El País, Negocios, Año XIV, no 818, 8 de Julio de 2001, pág. 5. 
unión con Dragados Inmobiliaria que se tradujo en un incremento de los beneficios en los seis primeros meses del 2001 de 35,1 millones de euros, que representa nada menos que un 71 por $100^{4}$.

Y todo este volumen de actividad está generado no ya como en el pasado por el promotor inmobiliario, sino por las grandes empresas promotoras; un agente que ha eclipsado a quienes durante buena parte del proceso de desarrollo urbano contemporáneo en España tuvieron la responsabilidad casi absoluta sobre el proceso: el propietario del suelo y el constructor y que, paradojas del destino, desde la aprobación de la ley de Ordenación de la Edificación tiene que desarrollar su labor en un contexto cada vez más exigente, compitiendo, en lo relativo a la intermediación inmobiliaria, tras la aprobación del RD Ley 4/2000 sobre medidas urgentes de liberalización en el sector inmobiliario y del Transporte, en un contexto de liberalización extrema que exonera al intermediario de ofrecer cualquier garantía. La respuesta por parte del sector ha sido inmediata y consiste por lo general en integrar también esta etapa del proceso - la intermediación inmobiliaria - dentro de su organigrama ya sea creando una división específica o ya sea adquiriendo redes de intermediación inmobiliaria ya existentes y con amplia implantación territorial ${ }^{5}$. Y con ello alcanzan un doble objetivo: ampliar el escaparate, es decir, el territorio que se pone a la venta y eliminar la competencia local que, por conocer mejor el terreno, no había tenido hasta ahora prácticamente la competencia de las grandes empresas.

\section{Del promotor a la gran promotora inmobiliaria: la versatilidad de la Ley 38/1999, de 5 de Noviembre de Ordenación de la edificación}

De entre todos los agentes que más o menos regulados y con mayor o menor profesionalidad intervienen en el mercado inmobiliario-propietarios, promotores, constructores, intermediarios, la Administración etc... y frente al destacado papel que en otras épocas han jugado los propietarios o constructores, quizá uno de los que tienen en el momento presente mayor importancia sean los promotores inmobiliarios. Un promotor inmobiliario es la persona física o jurídica (incluidas las cooperativas de viviendas) que, con o sin ánimo de lucro, — generalmente con él- construye o encarga la construcción de viviendas para uso propio o de terceros, por cuenta propia o como mandataria, gestora o representante del dueño del suelo. Es un grupo muy heterogéneo, ya que de forma circunstancial o estructural puede ser promotores inmobiliarios, tanto los propietarios del suelo que realizan operaciones de promoción para si o para otros, como los promotores profesionales con dedicación preferente pero que ya no es exclusiva a esta labor, dada la importancia de otras actividades de gestión en la cartera de negocio de estas empresas, y los promotores-constructores, las entidades financieras, los grupos de empresas en principio ajenas al sector inmobiliario y obviamente el Estado cuando actúa como promotor de viviendas para los segmentos del mercado de menor poder adquisitivo ${ }^{6}$.

Un promotor - especialmente las compañías inmobiliarias - se ocupa de comprar terrenos, de encargar la construcción a otras empresas constructoras y de venderlos a terceros

\footnotetext{
4 Merrill Lynch eleva sus previsiones sobre las inmobiliarias españolas. Expansión Directo, 31 de Julio de

5 Ferrovial inmobiliaria adquiere Don Piso, el primer grupo de intermediación inmobiliaria en España. 4 de Julio de 2001.

6 Carrasco, A. y otros. Derecho de la construcción y de la vivienda. Ed. Dilex, Madrid 1998.
} 2001. 
por lo general - y si es posible — antes de finalizar la construcción. La mayoría de inmobiliarias cuentan con una importante reserva de suelo, comprado con suficiente antelación, que puede garantizar su actividad por unos dos o tres años y en la medida en que finalizan proyectos reponen esta reserva comprando más terrenos. Además, los terrenos que compran las inmobiliarias son en su mayoría no urbanos ya que «...se trata de comprar donde aún no se puede edificar pero donde previsiblemente las Autoridades Públicas permitirán hacerlo a medio plazo, tras las oportunas recalificaciones ${ }^{7}$. Se necesita por lo tanto disponer de una fuerte capacidad financiera - lo que explicaría la presencia de grandes grupos bancarios entre su accionariado- que les permita esperar algunos años hasta que sus terrenos alcancen la condición de edificables y —muy importante—, asumir con ciertas garantías el riesgo de que esto nunca ocurra.

Pero, pese a la trascendencia de esta labor y la responsabilidad económica que comporta, sorprendentemente ni a nivel estatal ni regional, el ejercicio de la promoción inmobiliaria no está sujeto a ningún tipo de requisito. No se exige titulación o colegiación, ni inscripción en registro administrativo alguno o la disponibilidad de un capital mínimo que sirva de garantías a terceros. Y es que, según el artículo 9 la Ley 38/1999, de 5 de noviembre de Ordenación de la Edificación, ley que intenta equilibrar la desmedida atención que se ha prestado en España al suelo frente a otros componentes o agentes del proceso constructivo, un promotor es «...aquella persona física o jurídica, pública o privada que, individual o colectivamente, decide, impulsa, programa y financia, con recursos propios o ajenos, las obras de edificación para sí o para su posterior enajenación, entrega o cesión a terceros bajo cualquier título» ${ }^{8}$. Tan alto grado de versatilidad, teniendo además en cuenta que dentro de esta categoría tienen cabida tanto el promotor-constructor como el promotor-vendedor, posibilita la intervención en este sector de personas o empresas que descuidan las obligaciones que la ley señala — art. 9.2- y generan, año tras año, el mayor número de quejas ante las oficinas de Defensa del Consumidor, o ante el Defensor del Pueblo en cada Comunidad Autónoma. Y el catálogo histórico de irregularidades comprende todo aquello que en la mencionada ley se traduce en obligación o responsabilidad; en efecto, en no pocas ocasiones el promotor «...no ostentaba sobre el solar la titularidad de un derecho que le faculte para construir en él»-9.2.a-; no siempre se «...facilita la documentación e información previa necesaria para la redacción del proyecto» —9.2.b-; en ocasiones no se gestionan y obtienen «...las preceptivas licencias y autorizaciones administrativas» -9.2.c-; era relativamente habitual que no se suscribiesen los seguros correspondientes -9.2.d-y por último no siempre se entrega «...al adquiriente, en su caso, la documentación de obra ejecutada o cualquier otro documento exigible por las Administraciones competentes.»9.2.e-9.

En cualquiera de los dos casos — constructores o vendedores- existe un elemento común y es el enorme peso específico que en la historia de la ciudad ha tenido la propiedad del suelo en el desarrollo de autopromociones o en la formación de sociedades-empresas dedicadas, de forma más o menos directa, a la construcción y la promoción. Es más, durante algunas décadas fue muy común que fuese el propietario de solares en algunos suburbios o

\footnotetext{
7 http://www.labolsa.com

8 Carrasco, A., Cordero, E. y González M.C. Comentarios a la ley de ordenación de la edificación. Aranzadi, Ed. Elcano (Navarra) 2001, pp. 653.

9 Ley 38/1999, de 5 de Noviembre. Ordenación de la edificación (BOE núm. 266, de 6 noviembre) Art. 9.2 $(\mathrm{a}, \mathrm{b}, \mathrm{c}, \mathrm{d}, \mathrm{e})$.
} 
núcleos extrarradio el que asumiese el riesgo de emprender la aventura empresarial, aprovechando el enorme déficit de vivienda, las escasas exigencias técnico-legales en esta materia, la inexistencia de planeamiento y la venta segura de todo lo que se ponía en el mercado; se trataba de promotores de fortuna, que no desarrollan muchas promociones al tiempo ni actúan por lo general más de un lustro ${ }^{10}$. Obviamente esta aventura duró relativamente poco, ya que, salvo en aquellos casos en los que se dispuso de un apoyo institucional, el final de la construcción coincidía con el cierre de la empresa creada ad hoc. Hasta los años cincuenta, la transformación de suelo rústico en urbano corrió a cargo por lo tanto de hacendados agrícolas — con profundas raíces en la estructura política de la Dictadura-, grandes comerciantes, industriales y profesionales liberales que invierten en fincas en la periferia de las ciudades y de también modestos propietarios que movilizan una porción de suelo, pequeña en cada promoción, pero relativamente importante en el conjunto ante la escasa profesionalización del sector y la limitada presencia todavía de empresas foráneas — de otras ciudades o provincias- ${ }^{11}$.

A partir de la entrada en vigor de la primera Ley del Suelo en 1956, coincidiendo con el inicio del éxodo rural casi sistemático y aprovechando las enormes ventajas que ofrecía la aplicación combinada de la ley sobre viviendas subvencionadas y el plan Nacional de la Vivienda de 1961, diversos promotores profesionales van asumiendo de forma progresiva la totalidad del proceso constructivo, desde la parcelación del suelo hasta su construcción directa. Los propietarios del suelo, que siguen apareciendo en la solicitud de parcelaciones, lo hacen asociados a los promotores profesionales, siendo habitual la cesión de suelo a cambio de viviendas o de porcentajes del beneficio extraído por la venta. Todavía en este periodo la mayor profesionalización no se traduce en una diversidad de formas, estructuras o en una mejora de la calidad de la edificación; las causas hay que buscarlas tanto en el bajo nivel de exigencia de la nueva demanda de vivienda en propiedad, como porque son relativamente escasos los estudios de arquitectura que actúan en cada ciudad, por lo que era habitual que se adaptase una y mil veces el mismo modelo que por otra parte estaba condicionado por la disponibilidad, calidad y precio de los materiales que se podían encontrar en el mercado ${ }^{12}$.

Este proceso de profesionalización del sector inmobiliario se apoyó en el cambio histórico del mercado de alquiler al mercado de construcción para la venta y de rehabilitación. El proceso de difusión de la propiedad horizontal como forma más extendida de la propiedad urbana, atrae al capital financiero al sector, de modo que en la medida en que aumenta el número de propietarios, estos pierden en capacidad de decisión, pasando ésta a manos de un agente, los promotores inmobiliarios, que dejan de limitarse a prestar tan sólo unos servicios temporales, para constituirse en entidades con alta capacidad para modelar el espacio con todas sus consecuencias, aprovechando, además, algunas coyunturas de carácter financieroinversor como es el papel que están empezando a desempeñar los fondos de inversión inmobiliaria $^{13}$.

10 García Cuesta, J.L. De la urgencia social al negocio inmobiliario. Promoción de viviendas y desarrollo urbano en Valladolid 1960-1992. Ayuntamiento y Universidad de Valladolid 2000, cfr. Pp. 148.

11 Villagrasa, J. (Coord). Vivienda y promoción inmobiliaria en España. Universidad. de Lleida 1997, 249 pp.

12 García Cuesta, J.L. De la urgencia social... Op. Cit. pp. 184.

13 Según la Comisión Nacional del Mercado de Valores, a 27 de Octubre de 2000 los cinco (5) fondos de Inversión Inmobiliaria, regulados por la Ley 19/1992 eran los siguientes BCH-BANIF Fondo inmobiliario, registrado el 21-02-1998, Eurofondo Propiedad (21-02-1995), Santander Inmobiliario (27-03-1998), Segurfondo Inmobiliario (21-02-1998) y Segurfondo Inversión (21-02-1995). 
De este modo puede afirmarse que ante la escasa cultura empresarial y la debilidad de otras áreas de negocio, la consolidación del sector se apoyó, de forma preferente en origen, en el acceso a la propiedad, en la compra de un producto cuyo precio solo de forma episódica ha descendido y que, por contra, viene incrementándose en los últimos años por encima del 10 por 100 hasta alcanzar el cenit en el año 2000 con un 16 por 100, es decir cuatro veces el IPC real y hasta ocho veces el IPC previsto por el gobierno en los últimos años ${ }^{14}$. Un crecimiento desproporcionado, que exige un gigantesco esfuerzo familiar para la adquisición de una vivienda ya que, a pesar de tener los tipos de interés más bajos de Europa, España es uno de los países donde el esfuerzo de una familia para adquirir una vivienda es superior al resto. De modo más concreto y como se puede apreciar en el cuadro ํํำ 1 , España es el cuarto país de los quince reseñados, donde una familia dedica más esfuerzo para la compra de la vivienda (un 34,4 por ciento), un 0,92 por ciento más que la media europea - ver cuadro $\mathrm{n}^{\mathrm{o}} 1$ - El país que menos esfuerzo dedica en la compra de la vivienda es Francia, con un 29 por ciento de la renta familiar, seguido de Bélgica, que dedica un 30 por ciento de la misma. En el lado opuesto se encuentra Grecia que, con un 40,5 por ciento, es el país en el que más esfuerzo se hace en la compra de la vivienda seguido de Portugal con un 36,1 por ciento $^{15}$. En una primera aproximación puede deducirse de este hecho que, si a pesar de tener los tipos de interés más bajos de Europa el esfuerzo familiar para adquirir una vivienda es de los más elevados, el problema debe encontrarse o bien en la menor renta familiar o bien - y esta es la hipótesis que sostenemos- en el mayor precio de las viviendas, hecho que explicaría el inusitado optimismo urbanizador, es decir, la fortaleza que en el primer año del siglo XXI caracteriza al sector de la construcción y promoción inmobiliaria en España.

Y tan desproporcionado esfuerzo de adquisición debido al elevado precio de la vivienda no puede imputarse exclusivamente a la falta absoluta de suelo, como habitualmente se hace, sino al escaso grado de movilización del mismo, por interés del binomio propiedadpromoción, por el escaso grado de elaboración del planeamiento de desarrollo y por la larga, compleja y técnicamente disuasoria tramitación urbanística, que convierte a todo el sistema en extremadamente ineficiente ${ }^{16}$.

14 El precio de la vivienda libre subió el 15,5\% en el segundo trimestre del año 2001 respecto al mismo periodo del año anterior alcanzando las 171.430 pts. por metro cuadrado construido, cifra que esconde grandes diferencias ya que frente a los 271.800 pts del País vasco o las 260.200 de Madrid, el precio por metro cuadrado en Extremadura fue de 83.300. El país, Lunes 27 de Agosto de 2001, pág. 51.

En el tercer trimestre de 2000, las condiciones de financiación permitían, a los hogares con un único salario medio, el acceso a una vivienda de 13,3 millones de pesetas siempre que su capacidad de pago no sobrepasara una tercera parte de los ingresos. Sin embargo, a los precios actuales, el acceso a una vivienda de tipo medio supone un grado de esfuerzo de casi el $40 \%$ del salario bruto. Esta situación presenta algunas diferencias regionales. Así, es en el País Vasco, Baleares, Cataluña y la Comunidad de Madrid, el esfuerzo para acceder a una vivienda es mayor, superando el $50 \%$ de los ingresos brutos de una familia con un solo salario, cuadro 3.2. Situación Inmobiliaria. Febrero 2001. Servicio de estudios del BBVA.

15 http://www.inmonoticias.com/inter/europa_viv.HTM

16 En todo caso no es la falta de legislación la responsable de este estado de cosas desde 1990 cabe citar al menos la Ley 8/1990 de 25 de Julio sobre Reforma del Régimen Urbanístico y Valoraciones del Suelo. El Real Decreto-Legislativo 1/1992, de 26 de Junio, por el que se aprueba el texto refundido de la ley sobre el Régimen del Suelo y Ordenación urbana. -BOE 30.06.92—, el Decreto-ley 5/1996, de 7 de Junio sobre medidas liberalizadoras en materia de suelo -B.O.E. 8 Junio 1996- la) Sentencia 61/1997 de 20 de Marzo de 1997 del Tribunal Constitucional, la Ley 7/1997 de 14 de Abril sobre medidas liberalizadoras en materia de suelo —B.O.E. de 15 de Abril—, la Ley 6/1998 de 13 de Abril sobre Régimen del Suelo y Valoraciones. Finalmente a partir de 1998 se han ido aprobando leyes regionales completas como la Ley 5/99 de 8 de Abril de urbanismo de Castilla y León, o modificaciones a las medidas transitorias que fueron adoptando las CC.AA tras la sentencia del Tribunal constitucional. 
Cuadro 1

TIPO DE INTERÉS HIPOTECARIO Y \% DEL SALARIO QUE SE DEDICA A LA COMPRA DE UNA VIVIENDA (PRIMER SEMESTRE DE 2001)

\begin{tabular}{|l|c|c|}
\hline País & $\begin{array}{c}\text { Interés } \\
\text { hipotecario }\end{array}$ & $\begin{array}{c}\text { Esfuerzo familiar } \\
\text { (\% salario) }\end{array}$ \\
\hline Alemania & 5,78 & 34 \\
Austria & 6,24 & 34,8 \\
Bélgica & 6,58 & 30 \\
Dinamarca & 6,8 & 33,2 \\
España & $\mathbf{5 , 6}$ & $\mathbf{3 4 , 4}$ \\
Finlandia & 5,87 & 32,6 \\
Francia & 6,96 & 29 \\
Grecia & 7,02 & 40,5 \\
Holanda & 5,99 & 33,3 \\
Inglaterra & 6,9 & 32,4 \\
Irlanda & 5,98 & 31,5 \\
Italia & 6,88 & 34,9 \\
Noruega & 6,9 & 32,6 \\
Portugal & 6,4 & 36,1 \\
Suecia & 6,4 & 33 \\
\hline Media europea & 6,42 & 33,48 \\
\hline
\end{tabular}

Fuente: Asociación Hipotecaria Española y Banco Central Europeo.

Según señala J.R. Gancedo, e n los 3.320 municipios que a finales de 1992 tenían plan en España se desarrollaba como media «un plan parcial cada 8 años» y «...el tiempo total acumulado que viene siendo necesario en España para transformar el suelo en solar edificable, y por tanto para poder ofertar al mercado dicho solar, oscila, como medias reales, entre un mínimo de 83 meses y un máximo de 253 meses. Ni más ni menos que un mínimo de siete años y un máximo superior a 20 años $^{17}$. Con la particularidad añadida de que la promoción residencial está en manos básicamente de promotoras muy pequeñas, de implantación local, que no tienen reserva de suelo y que tienen que adquirirlo a propietarios o grupos inmobiliarios mayores, en una fase ya muy avanzada de la larga tramitación administrativa del mismo, entre 7 y 10 o más años y cuando está tan sólo a falta de la licencia; y este tipo de suelo es extraordinariamente caro, ya que, al elevado precio que se pagó por él en origen, hay que añadir el sobreprecio por los muchos años de reserva primero, por la escasez de solares después y el por los costes de urbanización y gestión finalmente, encareciéndose de forma desproporcionada el producto final. $Y$ en estas condiciones, nadie quiere promover viviendas protegidas a $100.500 \mathrm{pts} / \mathrm{m}^{2}$, simplemente porque «no salen las cuentas». Pero también es responsable de la situación actual la propia administración municipal ya que es a ella a la que compete la oferta de un suelo sometido a 17 normativas regionales diferentes; es sabido, en efecto, que el precio final de una vivienda tiene una enorme resistencia a la

17 Gancedo, J.R. «La crisis del sistema de planeamiento» en VV.AA., Perspectivas demográfico-sociales, urbanísticas y territoriales en el umbral del siglo XXI. Eunsa. Pamplona 1995, págs. 361-379. 
baja debido al control que los ayuntamientos ejercen sobre el precio del suelo ya que las subastas de suelo público — que constituyen una parte esencial de su financiación anualhan elevado el precio del suelo hasta en un 51,4 por 100 con respecto al precio de salida ${ }^{18}$.

En los últimos años, la promoción no residencial está siendo tan importante como la tradicional promoción de viviendas; una actividad emergente, que aprovecha el espectacular alza del precio de alquiler de oficinas y naves industriales y que ha llevado a alguna de estas empresas - por ejemplo a Inmobiliaria Colonial—a especializarse casi de forma exclusiva en construcción o la gestión de edificios de oficinas en alquiler en Barcelona y Madrid, ciudad que ocupaba en el año 2001 con 42,5 euros $/ \mathrm{m}^{2}$ el quinto lugar de Europa en lo relativo a los precios de alquiler de las mismas tras Londres - 98,9 euros $/ \mathrm{m}^{2}$ - París - 63,1 euros $/ \mathrm{m}^{2}$ - , Dublín — 47 euros $/ \mathrm{m}^{2}$ — y Frankfurt con 43,5 euros $/ \mathrm{m}^{2}{ }^{19}$. Se puede deducir de ello que el atractivo de este sector se ha consolidado de forma espectacular, olvidando pasadas crisis como la del final de los años setenta o la de los primeros años del noventa; y ello ha redundado en una presencia creciente de agentes más o menos profesionales en él, así como en una decidida apuesta por las fusiones o adquisiciones, en una diversificación de actividades, en una mejora de la implantación en el exterior y en un explosivo desarrollo de nuevas estrategias de venta - B2B y B2C - a través de internet; y es que, ciertamente, la penetración del ebusiness en nuestro país es todavía incipiente. Las transacciones on line en los portales inmobiliarios, tanto en venta electrónica a usuario final $-\mathrm{B} 2 \mathrm{C}-$ como en comercio entre empresas a través de Internet - B2B - se están desarrollando con un retraso de dos años respecto a la media europea y con tres respecto a la estadounidense ${ }^{20}$.

Finalmente, cabe destacar que, en los últimos años del siglo XX, una buena parte de las grandes empresas inmobiliarias han reducido el peso de la actividad básica —e históricacomo es la promoción residencial, en favor de un mayor control de las plusvalías que genera el proceso urbanizador por medio de la formación de bolsas de suelo urbanizable para su ulterior promoción y construcción, o merced a la compra y ulterior gestión de inmuebles para destinarlos al muy lucrativo mercado de alquiler de oficinas, parques empresariales, o de gestión de centros comerciales, centros de ocio o de ambos. Con todo, cada vez es más evidente que las empresas de mayor tamaño se han especializado en la compra estratégica y anticipada de suelo cuando todavía quedan algunos años y un largo proceso administrativo para su puesta en uso. Pero para llegar a este nivel, es decir, para aumentar su capacidad de control del proceso inmobiliario, para ampliar su área de influencia y para mejorar su competitividad, el sector inmobiliario ha tenido que adaptarse a las nuevas coyunturas impuestas por el mercado; y ha tenido que hacerlo en muy poco tiempo.

18 Las inmobiliarias, en la cresta de la ola. op. cit., pág. 5. Asimismo y como muestra de este hecho, baste señalar que el 25 de febrero de 1999 El Ayuntamiento de Valladolid adjudicó a la promotora Edificasa 2000 por 711 millones de pesetas una parcela de $1.223 \mathrm{~m}^{2}$ con una edificabilidad de $7.631 \mathrm{~m}^{2}$ valorada inicialmente en 155 millones, tras una subasta a la que concurrieron 19 empresas; en un solo acto público — una subasta- el suelo multiplicó su precio de salida — que ya era elevado- nada menos que 4,5 veces. El Norte de Castilla 25 de Febrero de 1999.

19 http://www.inmonoticias.com

20 Gran parte de los portales inmobiliarios son plataformas de negocios que abarca los tres niveles clásicos en el e-comerce: $\mathrm{C} 2 \mathrm{C}$-consumer to consumer-dirigido a particulares que pueden dar de alta o en su caso encontrar ofertas de compra o alquiler de todo tipo de inmuebles, un segundo nivel, B2C — business to consumer-, está dirigido a las empresas del sector inmobiliario para que puedan ofrecer a los usuarios - generalmente mediante sistemas de cuotas - sus promociones y ofertas inmobiliarias y finalmente el tercer nivel el es B2B - business to business - un punto de encuentro para hacer negocios entre todas las empresas del sector inmobiliario. 


\section{Fusiones, adquisiciones de empresas como estrategia de las grandes promotoras} inmobiliarias en España en los últimos años del siglo XX

A partir de 1995, las empresas inmobiliarias imprimen un cambio a la habitual estrategia del negocio inmobiliario, con objeto de ir adquiriendo nuevamente posiciones estratégicas en el sector. Los rasgos de este último periodo están sin duda presididos por las compras y fusiones de empresas, avaladas frecuentemente por los grandes grupos bancarios, como fórmula para competir en un mercado progresivamente más abierto, más exigente y con un futuro inmediato más inestable; como primera consecuencia, las promotoras han reducido el peso relativo de su negocio tradicional, la promoción de vivienda, al tiempo que han aumentado el peso de una actividad más segura y rentable: la patrimonialista, es decir, la gestión de alquileres —especialmente de oficinas-. Las fusiones de las inmobiliarias Bami y Zabálburu, y la adquisición de Vellehermoso Renta por Prima Inmobiliaria, son los más relevantes, pero también tiene una importancia considerable, por el efecto demostración que representa, la adquisición por parte de Urbis de la división inmobiliaria de una gran empresa constructora como Dragados, o la presencia creciente de empresas constructoras en el sector de la promoción como es el caso de Ferrovial-Inmobiliaria, empresa que desarrolla su actividad en 22 ciudades y tres países ${ }^{21}$. E incluso la colaboración al 50 por 100 entre una entidad bancaria como Caja Madrid y una empresa Constructora, FCC, para crear Realia, una nueva empresa inmobiliaria de presencia todavía embrionaria en el sector, pero llamada a jugar un papel destacado en el futuro. En todo caso, sólo a partir de una posición consistente desde el punto de vista empresarial y financiero - lo que se consigue ganando tamaño- es posible ganar territorio, es decir entrar a competir en todas las Comunidades Autónomas, con el muy atomizado sector promotor local —a veces en alianza con él- a veces frente a él -ver cuadro $\mathrm{n}^{\mathrm{o}} 2$ - .

Cuadro 2

ALGUNAS COMPRAS-FUSIONES RECIENTES EN EL SECTOR DE LA PROMOCIÓN INMOBILIARIA. AGOSTO 2001

\begin{tabular}{|l|l|l|l|}
\hline Empresa A & Operación & Empresa B & Nueva empresa/nueva actividad \\
\hline Urbis & $\begin{array}{l}\text { fusión por } \\
\text { absorción de }\end{array}$ & $\begin{array}{l}\text { División inmobiliaria } \\
\text { de Dragados }\end{array}$ & Urbis \\
\hline Prima Inmobiliaria & adquiere & Vallehermoso Renta & Testa/ alquiler de inmuebles \\
\hline Ferrovial Inmobiliaria & adquiere & Don Piso & Ferrovial-Don Piso \\
\hline Bami & $\begin{array}{l}\text { fusión por } \\
\text { absorción de }\end{array}$ & $\begin{array}{l}\text { Zabálburu e Inmopark 92 } \\
\text { Madrid }\end{array}$ & Bami \\
\hline FCC & crea al 50\% & Caja Madrid & Realia \\
\hline Metrovacesa & adquiere & Gesinar renta & $\begin{array}{l}\text { Metrovacesa-Gesinar } \\
\text { (división patrimonio) }\end{array}$ \\
\hline Grupo Lar & $\begin{array}{l}\text { firma una joint } \\
\text { venture con }\end{array}$ & Grosvener & $\begin{array}{l}\text { Explotación oficinas y centros } \\
\text { comerciales }\end{array}$ \\
\hline Grupo Lar & se asocia con & Ballesol & Explotación centros 3 ${ }^{\text {a }}$ Edad \\
\hline
\end{tabular}

Elaboración propia.

21 http://www.ferrovial.es 
Pero, aunque el esfuerzo resulta todavía insuficiente, ya que la cuota de mercado de las seis grandes empresas del sector — Vallahermoso, Metrovacesa, Urbis, Bami, Inmobiliaria Colonial y Filo - apenas representa el 5 por 100, comienza ya a ser notoria la expulsión del mercado de muchos pequeños promotores que no pueden resistir la presión de los grandes, aunque esta expulsión acaba resultando especialmente gravosa para casi todas las partes: en primer lugar para la gran empresa inmobiliaria, que tiene que hacer un gran desembolso para eliminar la competencia de las empresas - o uniones temporales de empresas- locales y adquirir los suelos que salen a subasta en ciudades en las que se quiere penetrar; y obviamente para el comprador final que tendrá que hacer frente a viviendas u oficinas más caras no sólo en esa promoción, sino en general en todas, por el efecto mimético que en calidades, precios y márgenes de beneficio tiene la actuación de una gran inmobiliariaconstructora. Y el mayor beneficiado de este sobreesfuerzo inversor es el propio Ayuntamiento quien, ante las dificultades económicas casi crónicas y la falta de una adecuada estructura financiero-presupuestaria, tiene que acudir a este procedimiento como fórmula para allegar recursos a un presupuesto siempre parco para unas ciudades con una demanda de servicios creciente.

Los procesos de fusión o absorción de empresas desarrollados en los últimos años son numerosos, destacando sobre ellos la fusión por absorción de Dragados Inmobiliaria por parte de Urbis, empresa participada por Banesto, para convertirse en la segunda inmobiliaria del país por cifra de negocio con un capital social de 127,6 millones de acciones y una valoración bursátil de 570,4 millones de euros o lo que es lo mismo 94.907 millones de pesetas. Asimismo, con fecha de enero de 2001 la compañía fusionada disponía de un banco de suelo de 2,8 millones de $\mathrm{m}^{2}$ edificables sobre rasante y más de un millón de $\mathrm{m}^{2}$ sobre rasante en proceso de construcción ${ }^{22}$. La necesidad de posicionarse en el futuro mercado inmobiliario es una de las razones que impulsa tal proceso, pero no ha sido la única razón; en el año 2001 se aprovecha todavía un momento en el que la demanda se sostiene por razones demográficas - hasta el 2004 - y porque en los próximos años todavía cabe esperar un aumento de la demanda por efecto del llamado esponjamiento de hogares, — más cantidad, menos tamaño- así como por los bajos tipos de interés.

En estas condiciones la fusión representa, además de considerables mejoras en el apartado financiero, una reducción del riesgo como consecuencia de la diversificación geográfica y la amplia gama de productos en oferta, ya que le permite afrontar en mejores condiciones las oportunidades de crecimiento futuro del sector. En efecto, es conocido que el negocio promotor es una actividad que tiene un elevado componente local, por lo que cualquier operación de fusión o adquisición debería servir para aumentar el número de lugares —regiones y ciudades - en los que se opera; la incorporación de Dragados Inmobiliaria a Urbis amplían la presencia de Urbis a ciudades como Santander, Tarragona, La Coruña, Santiago, Huesca, Madrid, Alicante, Valencia, Cádiz, Málaga, Las Palmas y Tenerife ${ }^{23}$. Asimismo, como justi-

22 La escritura de la fusión se firmó en Madrid el 29 de Junio de 2001.

23 Se estima, tras la fusión Urbis-Dragados, un crecimiento orgánico en términos de cifra de negocio por encima del 15 por 100 anual. En 2003 se prevé en la Promoción una cifra de negocio de 100.000 millones de pesetas con un margen bruto de 21.000 millones, en alquileres 7.500 millones de pesetas con un margen bruto de 5.500 millones y en rotación de suelo y patrimonio de 6.000 millones de pesetas con un margen bruto de 3.000 millones. Como consecuencia en 2003 los márgenes totales previstos son de 29.500 millones de pesetas con un beneficio neto estimado de 14.000 millones de pesetas. Presentación de la operación de fusión por absorción de Dragados Inmobiliaria S.A. por Inmobiliaria Urbis (Sociedad absorbente) Instituto de Analistas Financieros, Salón de Actos de la Bolsa De Madrid 28 de Marzo de 2001. 
ficante de la absorción se valora la escasez de superficie alquilable para oficinas en Madrid y Barcelona, que ha provocado en en los años 1999 y 2000 un espectacular alza en el precio de los alquileres y ha hecho aumentar el número de proyectos en ambas ciudades por un total de $800.000 \mathrm{~m}^{2}$ en 1999 y más de un millón de $\mathrm{m}^{2}$ en el año 2000.

Cuadro 3

INCREMENTO DE BANCO DE SUELO COMO CONSECUENCIA DE LA FUSIÓN POR ABSORCIÓN DE DRAGADOS INMOBILIARIA POR PARTE DE URBIS

\begin{tabular}{|l|c|c|c|}
\hline Actividad $\left(\mathbf{m}^{\mathbf{2}}\right)$ & Urbis & Dragados Inmobiliaria & Total \\
\hline Stock de suelo & 2.400 .000 & 418.000 & 2.818 .000 \\
Promociones en curso & 828.000 & 208.000 & 1.036 .000 \\
\hline Total & 3.228 .000 & 626.000 & 3.854 .000 \\
\hline
\end{tabular}

Fuente. Instituto de Analistas Financieros, Salón de Actos de la Bolsa de Madrid 28 de Marzo de 200124.

El 24 de Enero de 2001 culminó otra de las grandes y recientes operaciones de integración-fusión de dos grandes compañías del sector: Prima Inmobiliaria y Vallehermoso Renta, para crear Testa Inmuebles en Renta S.A., participada en un 64,96 por la primera y quedando el resto del capital en manos de los accionistas de Prima ${ }^{25}$; una operación que culmina poco más tarde, en Febrero, tras lanzar Vallehermoso una OPA que le lleva a controlar el 99,2 por 100 del capital de Testa, cotizando en bolsa el 0,8 por 100 restante del capital social. De este modo, la nueva sociedad optimiza todo el capital y experiencia de la antigua Prima Inmobiliaria y convierte a la nueva compañía en uno de sus tres pilares de actividad: Testa dedicada al alquiler de inmuebles — con más de un millón de $\mathrm{m}^{2}$ de inmuebles en explotación- Valoriza, que es la división de servicios y Vallehermoso división Promoción que conservaría la porción de actividad clásica de la promotora. Un cambio importante en una de las empresas con mayor tradición en el sector que además empezó rápidamente a ofrecer resultados positivos; en el primer semestre del año 2001 Vallehermoso obtuvo un beneficio neto de 69 millones de euros - 11.552 millones de pesetas_, un 28 por 100 más que el mismo período del año anterior ${ }^{26}$. Con todo ello, Vallehermoso, grupo participado en la actualidad por el BSCH, se ha consolidado como el primer grupo inmobiliario del país y una de las sociedades más atractivas para los inversores bursátiles. A 31 de Diciembre del año 2000 sus activos estaban valorados en medio billón de pesetas y el número de empleados se acercaba al medio millar $\_497-^{27}$. En su actividad promotora, Vallehermoso ha Urbis.

24 Presentación de la operación de fusión por absorción de Dragados Inmobiliaria S.A. por Inmobiliaria

25 Prima Inmobiliaria S.A. era una compañía inmobiliaria constituida el 1 de enero de 1974 cuya actividad era la adquisición de inmuebles para su arrendamiento, y otras actividades inmobiliarias en general. Su capital social alcanzaba antes de la fusión los 243 millones de euros y contaba con 45 inmuebles en rentabilidad fundamentalmente en Madrid y Barcelona. Las actividades de la compañía se centraban en el mercado español, con un $87 \%$ de sus inversiones, con una diversificación por subsectores: oficinas, industrial, comercial, y por arrendatarios, con más de 250 en la actualidad. La estructura de la compañía está concentrada en un reducido equipo de profesionales - 19 en 1999 — por lo que el grado de subcontratación es muy alto y afecta a todas las áreas corporativas no directamente relacionadas con el negocio inmobiliario. Protocolo para la integración de las actividades de renta de Vallehermoso S.A. y Prima Inmobiliaria S.A. Madrid, 13 de Julio de 2000 y CNMV.

26 http://www.vallehermoso.es

27 Fuente: CNMV: Los resultados se expresan en pesetas. 
continuado con la diversificación geográfica de sus promociones, como ya vienen haciendo por otra parte el resto de los grupos inmobiliarios,; a las ciudades en las que ya se tenía presencia antes del año 2000 como Madrid, Barcelona, Málaga, Sevilla, Alicante, Valencia, Santander, Ciudad Real, A Coruña, Tenerife, Oviedo, Bilbao, Córdoba y Palma de Mallorca se han añadido otras en el despliegue territorial de la política activa de compra de suelo: Cádiz, Granada, Lleida, Badalona, Girona, Valladolid, Salamanca, Tarragona, Ávila, Albacete, Murcia y Las Palmas ${ }^{28}$.

\section{Un cambio de estrategia: de la promoción residencial de carácter local a la gestión de alquileres de oficinas y SBA — superficie bruta alquilable- en centros comer- ciales a escala regional y nacional}

Como ya hemos señalado, el negocio inmobiliario clásico, es decir, la promoción de viviendas y oficinas para la venta dejó progresivamente paso, en los últimos años, a una escalada de diversificación, destacando en este proceso la actividad patrimonialista, es decir, la compra de activos para alquiler o gestión, especialmente de centros comerciales, oficinas, residencias o aparcamientos, ya que de ellos se obtienen rentas elevadas y seguras, a la vista de la favorable coyuntura de estas nuevas áreas de negocio, a saber: mayor demanda hotelera, mayor demanda de residencias de $3^{\text {a }}$ edad y mayor demanda de aparcamientos en un sistema que anualmente consume cerca del millón de nuevos vehículos ${ }^{29}$. Es también relevante la presencia creciente en el sector de los servicios a la edificación - del que es un modelo el grupo Vallehermoso y la creación de fondos inmobiliarios, pero es sin duda el mercado de alquiler para oficinas y la explotación de centros comerciales el que se está revelando como el más dinámico y estable para las empresas inmobiliarias, ya que el mercado de alquiler para viviendas es poco atractivo porque el porcentaje de viviendas alquiladas en España no llega al 15\% del total, y en su inmensa mayoría están en manos de particulares ${ }^{30}$.

Obviamente, esta actividad tiene un elevado carácter cíclico y aunque es comprensible que si la economía no va bien haya menos empresas dispuestas a alquilar instalaciones, el menor esfuerzo que supone un alquiler frente a una compra - no sólo en cantidad de dinero sino en menor rigidez de los compromisos para el arrendatario- hacen de ella una actividad más estable y con ingresos más predecibles. Pero, además de la señalada, existen otras causas que explican el que la mayoría de las inmobiliarias españolas esté desplazando el grueso de su negocio hacia esta actividad; la adopción de medidas fiscales destinadas a incentivar la opción del alquiler frente a la de la compra y las subastas de suelo condicionadas a la dedicación a alquileres durante una serie de años de los inmuebles construidos, parecen ser algunas de las razones que podrían explicar el importante crecimiento —en algunas compañías como Colonial o Metrovacesa- de la construcción y gestión de edificios destinados al alquiler de oficinas. Y este hecho no deja de tener también una notable incidencia urbanística ya que, por exigencias del mercado y a causa de las cada vez mayo-

28 http://www.vellehermoso.es

29 El relanzamiento de Grupo Filo está basado en una estrategia de claro posicionamiento de liderazgo en el mercado de centros comerciales. A cierre del ejercicio 1999 la superficie comercial de los centros en explotación ascendía a $210.831 \mathrm{~m}^{2}$ lo que supone una superficie bruta arrendable de $109.785 \mathrm{~m}^{2}$ repartida en cuatro centros comerciales. La composición de facturación ha experimentado un cambio importante, ya que en 1999 más del $50 \%$ de la facturación del Grupo deriva de ingresos por arrendamientos en comparación con el $40 \%$ del año anterior.

$30 \mathrm{http}: / / \mathrm{www} .1 \mathrm{abolsa} . \mathrm{com} / \mathrm{canales} / 276 /$ 
res exigencias en equipamiento y servicios complementarios, las inmobiliarias rotan periódicamente su patrimonio inmobiliario, es decir, venden los inmuebles más antiguos —que generan unos ingresos por alquileres menores - y compran o remodelan inmuebles en mejores condiciones, que además se encuentren situados en mejores zonas con lo que los ingresos para la empresa inmobiliaria son mayores.

Una de las actividades esenciales para entender el negocio inmobiliario en la actualidad es, sin duda, la promoción gestión o explotación de la Superficie Bruta Alquilable - SBAen los centros comerciales y de ocio que en los últimos años han ido inaugurándose en España. Frente a los 33 centros que se encontraban abiertos en 1980, al finalizar el año 2000 se encontraban ya en explotación nada menos que 413 centros comerciales con una superficie comercial de 6,7 millones de metros cuadrados y 440.000 plazas de aparcamiento, estando instalados en ellos 23.000 locales comerciales ${ }^{31}$. Y lo que es aún más relevante, en los seis primeros años del siglo XXI está prevista la instalación de otros 181 centros, cuyos proyectos se encuentran en diversa fase de tramitación, ejecución, lo que da idea más que suficiente de la importancia estratégica de esta actividad para las divisiones de renta o patrimonialistas de las promotoras inmobiliarias. Los principales proyectos previstos para este periodo siguen explotando el mismo modelo, aunque en algunos aspectos parece haberse producido cambios relevantes; la combinación de hipermercado y cine ya no es la locomotora del centro y su papel está siendo reemplazado por oferta de ocio, restauración, hoteles, destacando el interés de algunas discotecas, boleras y cadenas de gimnasios y fitness que demandan nada menos que $120.000 \mathrm{~m}^{2}$ durante los próximos años ${ }^{32}$.

El papel de locomotora lo representa también el propio emplazamiento, como sucede con las estaciones de ferrocarril — proyecto Vialia-Riofisa - o las zonas portuarias, o actividades decididamente lúdicas al margen de la oferta cinematográfica — pistas de hielo_-;

Cuadro 4

NÚMERO DE CENTROS COMERCIALES ABIERTOS EN LA DÉCADA DE LOS AÑOS NOVENTA $Y$ SUPERFICIE BRUTA ALQUILABLE

\begin{tabular}{|c|c|c|}
\hline Año & Centros Comerciales abiertos & SBA $\left(\mathbf{m}^{2}\right)$ \\
\hline 1991 & 23 & 296852 \\
1992 & 24 & 401349 \\
1993 & 34 & 625531 \\
1994 & 20 & 347065 \\
1995 & 26 & 622696 \\
1996 & 20 & 383073 \\
1997 & 19 & 477330 \\
1998 & 30 & 519388 \\
1999 & 16 & 222863 \\
2000 & 20 & 580000 \\
\hline TOTAL & 232 & 4476149 \\
\hline
\end{tabular}

Fuente: AEDECC (Asociación española de centros comerciales).

31 El Mundo, viernes, 11 de Mayo de 2001.

32 http://www.porlared.com 
Cuadro 5

LOS PRINCIPALES CENTROS COMERCIALES PROYECTADOS PARA EL PERÍODO 2001-2006

\begin{tabular}{|c|c|c|c|c|c|}
\hline Centro & $\operatorname{SBA}\left(\mathrm{m}^{2}\right)$ & Promotor & Centro & $\operatorname{SBA}\left(\mathrm{m}^{2}\right)$ & Promotor \\
\hline $\begin{array}{l}\text { Vialia estación } \\
\text { Málaga }\end{array}$ & 41000 & Riofisa/Vialia & $\begin{array}{l}\text { Gran Vía } \\
\text { (Hospitalet) }\end{array}$ & 32000 & LSGIE \\
\hline $\begin{array}{l}\text { Plaza Mayor } \\
\text { (Málaga) }\end{array}$ & 27990 & $\begin{array}{l}\text { Sonas/Castle } \\
\text { city }\end{array}$ & $\begin{array}{l}\text { Heron City } \\
\text { (Barcelona) }\end{array}$ & 22000 & Heron \\
\hline $\begin{array}{l}\text { Parque Victoria. } \\
\text { Rincón de la } \\
\text { Victoria (Málaga) }\end{array}$ & 41000 & Anjoca & $\begin{array}{l}\text { L`Anec Blau } \\
\text { Castelldefels }\end{array}$ & 36000 & $\begin{array}{l}\text { Comerciales de } \\
\text { Castelldefels }\end{array}$ \\
\hline $\begin{array}{l}\text { Marina del Parque } \\
\text { (Málaga) }\end{array}$ & 32000 & Chelverton & $\begin{array}{l}\text { Ponte Vella } \\
\text { (Orense) }\end{array}$ & 20000 & Ucosa \\
\hline $\begin{array}{l}\text { Parque Comercial } \\
\text { y de Ocio la } \\
\text { Algaida (Almería) }\end{array}$ & 53000 & GGC & Carrefour (Vigo) & 25000 & Carrefour \\
\hline $\begin{array}{l}\text { Parque Principado } \\
\text { (Oviedo) }\end{array}$ & 70000 & $\begin{array}{l}\text { Eroski- } \\
\text { Promodeico }\end{array}$ & Gran Vía de Vigo & 40000 & Promodeico \\
\hline $\begin{array}{l}\begin{array}{l}\text { La Carriona } \\
\text { (Avilés) }\end{array} \\
\end{array}$ & 16000 & Vallehermoso & $\begin{array}{l}\text { Príncipe Pío } \\
\text { (Madrid) }\end{array}$ & 43000 & Riofisa \\
\hline $\begin{array}{l}\text { Los Prados } \\
\text { (Oviedo) }\end{array}$ & 36000 & Carrefour & $\begin{array}{l}\text { Tres Aguas } \\
\text { (Alcorcón) }\end{array}$ & 60000 & Lend Lease \\
\hline $\begin{array}{l}\text { Festival Park } \\
\text { (Mallorca) }\end{array}$ & 50000 & $\begin{array}{l}\text { Festival } \\
\text { Park/DTZ }\end{array}$ & $\begin{array}{l}\text { La Vega } \\
\text { (Alcobendas) }\end{array}$ & 23000 & THI \\
\hline $\begin{array}{l}\text { Siete Palmas } \\
\text { (Las Palmas) }\end{array}$ & 50000 & $\begin{array}{l}\text { Inmobiliaria } \\
\text { Betancor }\end{array}$ & $\begin{array}{l}\text { La Dehesa } \\
\text { (Alcalá) }\end{array}$ & 9300 & Bouygues \\
\hline $\begin{array}{l}\text { El Muelle } \\
\text { (Las Palmas) }\end{array}$ & 45000 & Riofisa & $\begin{array}{l}\text { Espartales Sur } \\
\text { (Alcalá) }\end{array}$ & 10540 & Carrefour \\
\hline La Mareta (Telde) & 14000 & Canespen & $\begin{array}{l}\text { Araba Park } \\
\text { (Nanclares de } \\
\text { Oca- Álava) }\end{array}$ & 52700 & THI \\
\hline La Orotava & 30000 & $\begin{array}{l}\text { Alcampo } \\
\text { (LSGIE) }\end{array}$ & Lacua (Vitoria) & 17000 & Vallehermoso \\
\hline Carrefour (Tenerife) & 52000 & Carrefour & Ría XXI (Bilbao) & 21500 & Filo \\
\hline $\begin{array}{l}\text { Equinoccio } \\
\text { (Zaratán-Valladolid) }\end{array}$ & 60000 & Riofisa/Bami & Murueta (Durango) & 21255 & Eroski \\
\hline Vialia (Salamanca) & 11000 & Leclerc & $\begin{array}{l}\text { San Vicente del } \\
\text { Raspeig (Alicante) }\end{array}$ & 38000 & $\begin{array}{l}\text { Promodeico } \\
\text { /ING }\end{array}$ \\
\hline $\begin{array}{l}\text { Luz de Castilla } \\
\text { (Segovia) }\end{array}$ & 20100 & Gelco & $\begin{array}{c}\text { Bon hivern } \\
\text { (Alicante) }\end{array}$ & 40000 & $\begin{array}{l}\text { Alcampo } \\
\text { /LSGIE }\end{array}$ \\
\hline El Bulevar (Ávila) & 27000 & Manilex & $\begin{array}{l}\text { Puerta de Alicante } \\
\text { (Alicante) }\end{array}$ & 35000 & Carrefour \\
\hline $\begin{array}{l}\text { CC Talavera } \\
\text { (Toledo) }\end{array}$ & 18000 & Gad Gelco & $\begin{array}{l}\text { Vistahermosa } \\
\text { (Alicante) }\end{array}$ & 32000 & Chelverton \\
\hline $\begin{array}{l}\text { Diagonal Mar } \\
\text { (Barcelona) }\end{array}$ & 87000 & Hines & $\begin{array}{l}\text { Bonaire } \\
\text { (Aldaia-Valencia) }\end{array}$ & 80000 & Riofisa \\
\hline
\end{tabular}

Fuente: AEDEC. 
y no menos destacable es la capacidad de atracción que generan nuevas locomotoras comerciales como son los outlets - Main Outlet, La Roca o Factory- en los grandes centros de Madrid y Barcelona; el outlet es la venta de ropa de marca de calidad que, dentro de la temporada, sale al mercado un treinta por 100 más barata como fórmula para que los modistos y fábricas vendan sus excedentes y renueven su muestrario. La primera tienda outlet en España fue la cadena Main Outlet que abrió sus puertas en 1995; la Roca Company Stores se instaló poco después en Barcelona y Madrid - Las Rozas- y finalmente Factory opera en centros comerciales de Las Rozas y Getafe. En conjunto, desde 1995 se han instalado ya en España ocho Factory Outlet Center con un total de $85.000 \mathrm{~m}^{2}$, estando prevista la apertura en los primeros años del siglo XXI de al menos otros doce establecimientos que añadirán otros $190.000 \mathrm{~m}^{233}$.

El modelo, que amenaza con reducir a un papel relativamente marginal - al menos por volumen de ventas - al comercio textil a pie de calle, se repite en todos los centros, cualquiera que sea la promotora - Metrovacesa, Filo, Riofisa etc...- - y cualesquiera que sean las actividades «locomotoras», es decir las que ocupan mayor superficie y se constituyen en referentes, casi en la imagen de marca del centro: grandes superficies -Eroski, Carrefour, Hipercort-, tiendas de bricolaje — Leroy Merlín-, Distribuidoras cinematográficas Lauren, Warner-, tiendas de deportes —Décimas, Decathlon-. A estas actividades se añaden, en una proporción y distribución perfectamente medida, lugares de ocio, restauración, complementos-regalos Tiendas de deportes y fundamentalmente franquicias o tiendas de cadena en el sector textil: Zara, Mango, Massimo Dutti, A. Domínguez, Purificación García, Roberto Verino, Hugo Boss, Cortefiel, Berska, Fosco, Pinkie, C\&A, Benetton, Sprinfield, y otras muchas más hasta completar en algunos casos dos centenares de locales con una oferta combinada de estrategia de empresa - de cada empresa - y del centro en lo relativo a periodos de rebaja, técnicas de fidelización etc... En todo caso, los centros de mayor tamaño, con mayor número y diversidad de establecimientos, que generalmente se encuentran alejados de la ciudad acaban por reemplazarla íntegramente; se constituyen en una ciudad abreviada, comprimida, que además es segura al haber eliminado alguno de los aspectos inconfesables asociados a la economía capitalista finisecular: no hay mendicidad, ni transeúntes - homeless - ni delincuencia, ni suciedad en los pasillos rotulados como calles y plazas, ni recogida de basura, ni zanjas abiertas en sus calles. Ninguno de los rótulos desentona en esta ciudad que - parodia de las viejas ciudades cerradas- abre de 10 a 22 y que, al menos en España, aspira a hacerlo además todos los días del año incluyendo los domingos y las otrora denominadas fiestas de guardar. Uno de los proyectos de reciente apertura — septiembre de 2001 — es Heron City, en Barcelona, un centro promovido por la inmobiliaria inglesa Heron International y la española Layetana, que se promociona como «la ciudad de la diversión» siendo esta actividad y no la compra la «locomotora» de esta microciudad que esta decorada con motivos de la cultura y carácter mediterráneo. Para diferenciarse de otras centros comerciales, lo mismo que las ciudades para diferenciarse y competir con otras ciudades, Heron City contará con espectáculos coreografiados de agua, música y luz, así como música en vivo, cuatro locales de ocio nocturno, un gimnasio, un hotel y 16 salas de cine de última generación.

Y estas condiciones aseguran una estabilidad y seguridad tan altas —en lo relativo a las condiciones de explotación de los locales-, que pone a cubierto a las empresas promotoras

33 Expansión, 20 de Enero de 2001 y diarioinmobiliario.com, viernes 31 de Agosto de 2001. 
de las coyunturas que afectaban a los sectores clásicos de esta actividad; los ejemplos de Metrovacesa, compañía inmobiliaria del grupo BBVA que es uno de los mayores operadores en el sector de centros comerciales, así como Filo y Promodeico ilustran a la perfección la entidad y significado de esta rama de la actividad patrimonialista — promoción de centros comerciales para alquiler de locales - que junto con el alquiler de oficinas, parques empresariales, edificios industriales y hoteles conforma la base del negocio en este sector. Metrovacesa, explota locales en los centros que se reseñan en el cuadro $\mathrm{n}^{\circ} 6$.

Cuadro 6

CENTROS COMERCIALES DEL GRUPO METROVACESA. AGOSTO 2001

\begin{tabular}{|l|c|c|l|l|c|}
\hline \multicolumn{1}{|c|}{ Centro/lugar } & $\begin{array}{c}\text { Fecha de } \\
\text { apertura }\end{array}$ & Superficie $\left(\mathbf{m}^{2}\right)$ & \multicolumn{1}{|c|}{$\begin{array}{c}\text { Operadores } \\
\text { locomotoras }\end{array}$} & $\begin{array}{c}\text { Área de } \\
\text { influencia }\end{array}$ & $\begin{array}{c}\text { Visitantes } \\
\text { millones/año }\end{array}$ \\
\hline Artea (Leioa) & 27041998 & 33.960 & $\begin{array}{l}\text { Arasca, y } \\
110 \text { locales }\end{array}$ & $\begin{array}{l}\text { Margen } \\
\text { derecha }\end{array}$ & 7,7 \\
\hline $\begin{array}{l}\text { El centro de la } \\
\text { Moraleja }\end{array}$ & 1041995 & 18.000 & $\begin{array}{l}105 \text { locales. Moda } \\
\text { restauración }\end{array}$ & $\begin{array}{l}\text { Zona Norte } \\
\text { de Madrid }\end{array}$ & 4,7 \\
\hline El saler & 1101995 & 46.000 & $\begin{array}{l}\text { Carrefour y } \\
160 \text { locales }\end{array}$ & 1.200 .00 hab. & 9 \\
\hline La Maquinista & 15062000 & 80.000 & $\begin{array}{l}\text { Carrefour, Cines } \\
\text { Warner y } \\
230 \text { locales }\end{array}$ & $\begin{array}{l}\text { Sta. Coloma } \\
\text { de Gramanet }\end{array}$ & $\begin{array}{l}14 \\
\text { Galerías Saldanha }\end{array}$ \\
\hline
\end{tabular}

Fuente: http://www.metrovacesa.es

Una estrategia similar en lo relativo a la explotación de centros comerciales es la que representa el grupo Filo ya que obtiene más del 50\% de los ingresos de arrendamiento; su margen de negocio se ha situado en 3.728 Millones de pesetas, de los cuales el $70 \%$ deriva del área de Centros Comerciales y el 30\% del área de Promoción Residencial. Este hecho confiere solidez a la cuenta de resultados ya que los ingresos procedentes del arrendamiento de Centros Comerciales son recurrentes y tienen baja volatilidad, mientras que la componente del área residencial permite aprovechar las fases alcistas del ciclo inmobiliario.

La promoción, o gestión de los centros comerciales y de ocio se constituye de este modo en una de las mayores alternativas al negocio de las inmobiliarias tanto de las que podemos denominar tradicionales — por la diversificación de sus áreas de negocio - como de aquellas promotoras especializadas - al menos en origen — en la explotación de estos centros. Si el modelo de las primeras lo constituye Vallehermoso, Metrovacesa, Urbis o Filo, un buen ejemplo de las segundas es sin duda el que representa el grupo Promodeico, grupo creado en 1976 y que ha estado especializado hasta el comienzo del siglo XXI sólo en la gestión completa de todo el proceso de promoción y explotación de centros y locales comerciales: la búsqueda de terrenos, estudios comerciales y financieros, redacción del proyecto, construcción, comercialización, comunicación y gestión; la relación de los 22 centros de estas características que en la actualidad están en explotación, pone en evidencia la entidad y 
diversidad geográfica de su actividad, que en los últimos años se ha completado con áreas de actividad de carácter mas tradiciones en el negocio inmobiliario — promoción de viviendas, hoteles, residencias, edificios multiusos etc...-.

Pero la actividad patrimonialista no ha sido la única que ha contribuido a transformar el perfil de actividad de las empresas del sector de la promoción inmobiliaria. Prácticamente todas las grandes empresas aspiran a dirigir una parte del proceso urbanístico aportando una parte sustancial del suelo que, adquirido con la adecuada antelación y mantenido en reserva especulativa, será la materia prima del futuro urbanístico de todas las ciudades, especialmente ahora que lo que se espera es un incremento de las formas de urbanización difusa - urban sprawl, smart grow, edge cities etc...- que requieren de ingentes superficies de suelo que ya no está en manos de la propiedad tradicional, sino que ha pasado —está pasando en cada momento - a manos de las promotoras que disponen de reservas de suelo millonarias en todas las comunidades autónomas, en casi todas las provincias y en casi todas las fases de tramitación urbanística, desde solares a suelo apto para urbanizar, o

Cuadro 7

SUPERFICIE BRUTA ALQUILABLE EN CENTROS COMERCIALES GESTIONADA POR EL GRUPO PROMODEICO

\begin{tabular}{|l|r|}
\hline Centro Comercial & SBA $\mathbf{~}^{2}$ \\
\hline Área comercial y recreativa de la estación de Chamartín (Madrid) & 15.000 \\
Centrofama (Murcia) & 13.000 \\
Mercacentro (Las Palmas) & 2.000 \\
Mercado central (Las Palmas & 3.500 \\
CC Alcobendas (Madrid) & 4.500 \\
CC Carrefour Alameda (Málaga) & 7.000 \\
Puerta de Toledo (Madrid) & 16.000 \\
CC Carrefour (Zaragoza) & 4.750 \\
CC Abarca (Pontevedra) & 12.500 \\
CC Parc Central (Tarragona) & 27.000 \\
CC Parque Principado (Oviedo) & 70.000 \\
CC Las Huertas (Palencia) & 18.000 \\
CC La Loma (Jaén) & 30.000 \\
CC Baricentro. Barberá del Vallés (Barcelona) & 33.000 \\
CC Ruta de la Plata (Cáceres) & 16.500 \\
CC La Ballena (Las Palmas) & 55.000 \\
CC El Ferial (Parla-Madrid) & 16.000 \\
CC El Saler (Valencia) & 53.000 \\
CC Jorge Juan (Valencia) & 5.400 \\
CC El Centro de la Moraleja. Alcobendas (Madrid) & 16.000 \\
CC Gran Vía (Alicante) & 34.000 \\
CC El Tormes (Salamanca) & 23.500 \\
\hline
\end{tabular}

Fuente: http://promodeico.com. Elaboración propia. 
incluso de suelo rústico protegido para abarcar toda la gama posible de demanda; en el año 2001 cualquier promotora que aspire a estar presente en el negocio en los próximos años cuenta con un banco de suelo urbanizable o apto para urbanizar comprendido entre 2 y 4 millones de metros cuadrados. Que es mucho suelo y otorga mucho poder en la gestión en la sombra del urbanismo en España.

En conclusión, puestos los cimientos del negocio inmobiliario en un mercado - el mercado único- progresivamente más competitivo, y a pesar de que todavía en 2001 las viviendas se venden casi en su totalidad sobre plano y que hay una gran demanda de oficinas y de locales para alquilar en los grandes Centros Comerciales, las empresas inmobiliarias han comenzado a plantearse algunos cambios en las tradicionales estrategias de comercialización del producto; la competencia con agentes libres, en un mercado desregulado y en cierta medida desprofesionalizado es tan elevada, que los mayores grupos se están planteando participar también en los márgenes de un negocio, que cada vez son mayores por otro lado a la vista de la reducción de costes con que puede gestionarse el producto para su venta. $\mathrm{El}$ incremento de mercados verticales - $\mathrm{B} 2 \mathrm{C}$ - en internet, o de webs específicamente comerciales (e-comerce) o la estrategia de Ferrovial Inmobiliaria adquiriendo Don Piso, uno de los mayores grupos de intermediación inmobiliaria clásica en España, parecen ser alguno de los rasgos futuros del sector.

\section{Bibliografía}

CALDERÓN, B. (1998): Patrimonio residencial y ciudad tradicional en España al finalizar el segundo milenio. Universidad de Burgos, Burgos, pp. 107-120.

CARRASCO, A. y otros (1998): Derecho de la construcción y de la vivienda. Ed. Dilex, Madrid.

CARRASCO, A. y otros (2001): Comentarios a la ley de ordenación de la edificación. Aranzadi, Ed. Elcano. 848 pp.

ESCUDERO, A. (1996): Estudio del merciado inmobiliario español. Evolución, análisis y tendencias 1974-1996. CISS Gestión, Valencia, 330 pp.

ESCUDERO, A. (1995): Marketing inmobiliario 1. Métodos y técnicas de investigación. CISS, Valencia, 262.

ESCUDERO, A. (1995): Marketing inmobiliario 2. Métodos y técnicas de comercialización. CISS, Valencia, $289 \mathrm{pp}$.

GANCEDO, J.R. (1995): «La crisis del sistema de planeamiento» en VVAA. Perspectivas demográfico-sociales, urbanísticas y territoriales en el umbral del siglo XXI. Eunsa. Pamplona, págs. 361-379.

GARCÍA CUESTA, J.L. (2000): De la urgencia social al negocio inmobiliario. Promoción de viviendas y desarrollo urbano en Valladolid 1960-1992. Ayuntamiento y Universidad de Valladolid.

GARCÍA MONTALVO, J. y MÁS, M. (2000): La vivienda y el sector de la construcción en España. Caja de Ahorros del Mediterráneo. Valencia. 442 págs.

LÓPEZ, I. y otros (2000): Inversión y Gestión del patrimonio Inmobiliario. Cuadernos Cinco Días. Madrid.

MINISTERIO DE FOMENTO (1999): Edificación y vivienda 1993-1998. Datos recogidos de las licencias de obra concedidas por los ayuntamientos. Madrid.

VILAGRASA, J. (Coord). (1997): Vivienda y promoción inmobiliaria en España. Universidad de Lleida, 249 pp. 